6. Cardaillac, Claire. Surgical management of Bartholin cysts and abscesses in French university hospitals.Journal of gynecology obstetrics and human reproduction. 2019;48(8): 631-635.

7. Wechter ME. Management of Bartholin duct cysts and abscesses: a systematic review. Obstet
Gynecol Surv. 2009;64(6):395-404.

8. RotemR. Risk factors associated with recurrent referral to the emergency room following surgical treatment of Bartholin's gland abscess.Journal of Obstetrics and Gynaecology. 2020;40(1):111-115.

\title{
GIÁ TRỊ CỦA SIÊU ÂM KẾT HỢP ĐÀN HỒI MÔ TRONG CHẨN ĐOÁN UNG THƯ TUYẾN TIỀN LIỆT
}

\author{
Đoàn Thị Nga ${ }^{1}$, Nguyễn Lan Hương ${ }^{2}$, Nguyễn Duy Hùng ${ }^{1,2}$, \\ Lê Thanh Dũng ${ }^{2}$, Nguyễn Duy Huề
}

\section{TÓM TẮT}

Mục tiêu: đánh giá giá trị của siêu âm kết hợp đàn hồi mô trong chẩn đoán ung thư tuyến tiền liệt (UTTTL). Đối tượng và Phương pháp: 74 bệnh nhân nghi ngờ UTTTL được siêu âm qua đường trực tràng đa phương tiện gồm 2D, Doppler màu và $S E$, được đối chiếu với kết quả giải phâu bệnh qua sinh thiết hệ thống 12 mẫu dưới hướng dẫn siêu âm qua đường trực tràng tại bệnh viện Hữu nghị Việt Đức từ tháng $5 / 2020$ đến tháng $6 / 2021$. Kết quả: Nghiên cứu có $28 / 74$ bệnh nhân ung thư $(38 \%)$, phát hiện $78 / 296$ tổn thương ung thư ở 2 vùng chính là vùng ngoại vi (36) và vùng chuyển tiếp (42).Trong chẩn đoán UTTTL nằm ở vùng ngoại vi, siêu âm 2D, 2D+Doppler màu, 2D+Doppler màu+SE có độ nhay lần lượt là $72.2 \%, 72.2 \%, 80.6 \%$. Trong chẩn đoán UTTTL nằm ở vùng chuyển tiếp, siêu âm 2D, 2D+Doppler màu, 2D+Doppler màu+SE có độ nhạy lần lượt là 40.5\%, 66.7\%, 85.7\%. Kết luận: Siêu âm 2D qua đường trực tràng có độ nhạy cao trong chẩn đoán UTTTL nằm ở vùng ngoại vi nhứng có độ nhạy thấp trong chẩn đoán UTTTL nằm ở vùng chuyển tiếp. Kết hợp hai hoă̆c ba kĩ thuật siêu âm đều làm tăng độ nhay trong chẩn đoán UTTTL đặc biệt ở vùng chuyển tiếp.Siêu âm đàn hồi mô là phương pháp bố trợ giúp làm tăng đôn nhay trong chẩn đoán UTTTL.

Tư khóa: ung thư tuyến tiền liệt, siêu âm qua đường trực tràng, siêu âm kết hợp đàn hồi mô.

\section{SUMMARY}

\section{VALUES OF ULTRASOUND ASSOCIATED ELASTOGRAPHY IN THE DIAGNOSIS OF PROSTATE CANCER}

Purpose: This study aims to evaluate the value of ultrasound associated elastography in the diagnosis of prostate cancer. Material and Method: 74 patients with suspected prostate cancer was performed transrectal multiparametric ultrasound includes 2D,

\footnotetext{
${ }^{1}$ Trường đại học Y Hà Nội,

²Bệnh viện Hữu nghi Việt Đức

Chịu trách nhiệm chính: Đoàn Thị Nga

Email: Doannga0226@gmail.com

Ngày nhận bài: 4.8.2021

Ngày phản biện khoa học: 5.10.2021

Ngày duyệt bài: 14.10.2021
}

color Doppler and elastography, was compare with Histological results follow transrectal US guide system biopsy12 core at the Viet Duc Hospital from May 2020 to June 2021. Results: This study has $28 / 74$ patients with prostate cancer (38\%), detect 78/296 cancer lesions located in two main zones are peripheral zone (36) and transition zone (42). Diagnosis cancer in peripheral zone on 2D, 2D+color Doppler, 2D+color Doppler+SE has sensitivity of $72.2 \%, 72.2 \%, 80.6 \%$, respectively. Diagnosis cancer in transition zone on 2D, 2D+color Doppler, 2D+Doppler+SE has sensitivity of $40.5 \%, 66.7 \%, 85.7 \%$. Conclusion: Transrectal ultrasound $2 \mathrm{D}$ has high sensitivity in the diagnosis prostate cancer in the peripheral zone and low sensitivity in the diagnosis prostate cancer in the transition zone. To combine two or three technical ultrasound increase the sensitivity in diagnosis prostate cancer, especially in transition zone. Sonoelastography is supplement way to increase sensitivity in the diagnosis prostate .

Keywords: prostate cancer, transrectal ultrasound, ultrasound associated elastography.

\section{I. ĐẶT VẤN ĐỀ}

Ung thư tuyến tiên liệt (UTTTL) là loại ung thư thường gặp nhất và là nguyên nhân gây tử vong thứ tư do ung thư ở nam giới ${ }^{1}$. UTTTL có tỉ lệ sống sau 5 năm là $100 \%$ nếu được phát hiện sớm ở giai đoạn còn khu trú, tuy nhiên, ở giai đoạn đã có di căn, tỉ lệ này giảm xuống chỉ còn $32 \%$, do đó việc chẩn đoán sớm và điều trị kịp thời có vai trò rất quan trọng.

Siêu âm là phương pháp chẩn đoán cơ bản và đâuu tay trong chẩn đoán UTTTL nhưng hiện nay chưa có một kĩ thuật siêu âm riêng lẻ nào được coi là tối ưu, do vậy sinh thiết hệ thống dưới hướng dẫn của siêu âm qua đường trực tràng vẫn là phương pháp được lựa chọn để chẩn đoán xác định UTTTL ở những bệnh nhân có PSA> $4 \mathrm{ng} / \mathrm{ml}^{2}$. Siêu âm TTL qua đường trực tràng (TRUS) đã khắc phục được những nhược điểm của siêu âm qua đường bụng và là phương pháp chính trong phát hiện các tổn thương của TTL. TRUS bao gồm đồng thời siêu âm 2D và siêu âm Doppler, ngay nay còn được bổ sung thêm siêu 
âm đàn hồi mô (SE) đánh giá độ cứng của mô, được tích hợp trong cùng một đầu dò ${ }^{3}$.

Các mô bệnh lý có thể có cùng độ hồi âm nhưng chúng có độ cứng khác nhau, mồ càng ác tính thì độ cứng càng tăng. Siêu âm 2D không phát hiện được những tổn thương ung thư đồng âm. Nhiêu trường hợp tổn thương ung thư không tăng sinh mạch, vì vậy siêu âm Doppler vẫn khó nhận định tính chất lành tính hay ác tính ${ }^{4}$. Siêu âm đàn hồi mô đánh giá được độ cứng của mô tổn thương từ đó cung cấp thêm thông tin cho siêu âm $2 \mathrm{D}$ và Doppler trong chẩn đoán UTTTL ${ }^{5}$.

Cho đến nay, trên thế giới đã có nhiều nghiên cứu về ứng dụng của siêu âm kết hợp đàn hồi mô trong đánh giá các tổn thương khu trú tại $T T L$, cụ thể là khả năng phân biệt tổn thương lành tính hay ác tính. Tuy nhiên tại Việt Nam, kĩ thuật siêu âm đàn hồi vẫn chưa được ứng dụng rộng rãi và có rất ít nghiên cứu đề cập đến giá trị của siêu âm đàn hồi trong chẩn đoán UTTTL. Vì vậy chúng tôi tiến hành nghiên cứu này nhằm đánh giá giá trị của siêu âm kết hợp đàn hồi mô trong chẩn đoán UTTTL.

\section{II. ĐỐI TƯợNG VÀ PHƯƠNG PHÁP NGHIÊN CỨU}

Đối tượng nghiên cứu. Nghiên cứu được tiến hành trên 74 bệnh nhân được nghi ngờ UTTTL (thăm trực tràng nghi ngờ hoặc xét nghiêm $\mathrm{PSA}>4 \mathrm{ng} / \mathrm{ml}$ hoặc bằng các phương pháp CĐHA như siêu âm qua đường bụng, cộng hưởng từ), được siêu âm qua đường trực tràng kết hợp đàn hồi mô, được sinh thiết 12 mẫu và có kêt quả giải phẫu bệnh.

Kĩ thuật siêu âm. Thăm khám được thực hiện trên máy siêu âm LOGIQ S7 Expert với đầu dò trực tràng hai bình diện BE9CS có tần số từ 8 đến $10 \mathrm{MHz}$ và có kênh sinh thiết tích hợp đồng bộ.Bệnh nhân được giải thích quy trình, đi tiểu trước khi thực hiện siêu âm. Tư thế bệnh nhân: nằm nghiêng trái, hai chân co tạo góc giữa đùi và bụng nhỏ hơn hoặc bằng 90 độ. Khảo sát TTL một cách toàn diện trên các lớp cắt dọc và cắt ngang theo trình tự từ đỉnh đến đáy $T L$ và từ phải sang trái. Khảo sát tuần tự từ hình thái, huyết động đến tính chất vật lý: siêu âm 2D, siêu âm Doppler màu, siêu ẩm đàn hồi. Trong thu hình siêu âm đàn hồi, ROI cần được bao phủ toàn bộ tuyến và mô xung quanh nhưng không bao gồm bàng quang để tránh ảnh giả. Thực hiện ép đầu dò với lực thật nhe (mức dịch chuyển đâu dò khoảng $1 \%$ độ sâu của vùng mô chứa $T T L$ ) rồi nhả ép, thực hiện trên cùng mặt phẳng cắt. Ghi nhận hình ảnh sau khi nhả ép rồi lặp lại cho đến khi thu chọn được hình với chỉ điểm chất lượng đạt. Sau khi thu được một seri ảnh đàn hồi, sẽ chọn hình tối ưu là hình có yếu tố chất lượng (quality factor) cao nhất ${ }^{6}$. Toàn bộ dữ liệu ảnh và video đều được lưu trữ trên máy siêu âm.

Phân tích hình ảnh. Tiêu chuẩn chẩn đoán UTTTL trên siêu âm 2D gồm một trong các dấu hiệu sau: (1)Vùng ngoại vi: vùng có độ hồi âm thay đổi so với nhu mổ xung quanh \pm phá võ vỏ tuyến \pm xâm lấn nhu mô xung quanh \pm bờ tuyến không đều \pm tuyến mất cân đối, (2)vùng chuyển tiếp: vùng có độ hồi âm thay đổi so với nhu mô xung quanh và đẩy lồi về phía ngoại vi \pm bờ tuyến không đều \pm tuyến mất cân đối ${ }^{3}$.

Tiêu chuẩn chẩn đoán UTTTL trên siêu âm Doppler màu gồm một trong các dấu hiệu sau: tăng tín hiệu dòng chảy khu trú, tín hiệu dòng chảy không đối xứng tại mô ung thư cùng với tăng số lượng và kích thước mạch máu ${ }^{3}$.

Tiêu chuẩn chẩn đoán UTTTL trên siêu âm đàn hồi: phân tích hình ảnh trên bản đồ màu,sử dụng 5 thang điểm của Kamoi dựa vào mức độ và sự phân bố tính đàn hồi của mô tương quan với tổn thương giảm âm trên 2D. Hệ thống phân loại này mã hóa mô cứng là màu xanh dương và mô mềm là màu đỏ hoặc xanh lá.Score 1: bình thường (mật độ đồng nhất). Score 2: khả năng bình thường (mật độ không đồng nhất dạng khảm). Score 3: khổng xác định (ổ cứng - màu xanh không đối xứng và khồng liên quan với có một tổn thương giảm âm). Score 4: khả năng ác tính: có tổn thương giảm âm với cứng (màu xanh ở trung tâm và còn mềm ở ngoại vi). Score 5 : chắc chắn ác tính: có tổn thưởng giảm âm với cứng toàn bộ và cả tổ chức xung quanh. UTTTL được nghi ngờ khi mô tổn thương có score từ 3 trở lên ${ }^{7}$.

Tiêu chuẩn chẩn đoán UTTTL khi kết hợp 2 hoặc 3 kĩ thuâtt 2D, Doppler màu, đàn hồi: khi có ít nhất một tiểu chuẩn chẩn đoán UTTTL của một kĩ thuật siêu âm.

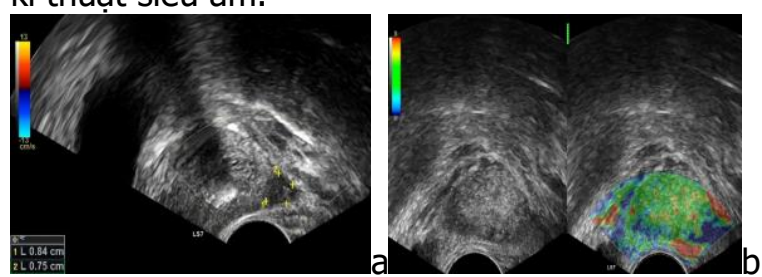

Hình 1: Bệnh nhân P.H.T 65 tuổi, PSA $7.89 \mathrm{ng} / \mathrm{ml}$, (a) tổn thương giảm âm vùng ngoại vi trái không tăng sinh mạch trên siêu âm $2 \mathrm{D}$ và Doppler màu, (b) tổn thương tương ứng $\mathrm{SE}$ score 4 trên siêu âm đàn hồi (trung tâm màu xanh dương biểu hiện cứng, ngoại vi màu xanh 
lá biểu hiện mềm), gleason 4+4=8.

Xử lý và phân tích số liệu. Số liệu được nhập và phân tích bằng phần mềm SPSS 20.0 (SPSS, Inc., Chicago, Hoa Kỳ). Các biến được trình bày dưới dạng số và tỷ lệ phần trăm.

Giá trị của siêu âm 2D, Doppler, đàn hồi và kết hợp ba phương pháp trong chẩn đoán xác định UTTTL được xác định bằng độ nhạy, độ đặc hiệu, giá trị dự đoán dương tính, giá trị dự đoán âm tính và độ chính xác với kết quả giải phẫu bệnh là tiêu chuẩn vàng.

\section{KẾT QUẢ NGHIÊN CỨU}

Từ tháng $5 / 2020$ đến tháng 6/2021, 74 bệnh nhân được nghi ngờ UTTTL (từ 55 đến 89 , độ tuổi trung bình là $67.22 \pm 6.96$, PSA toàn phần trung bình là $31.57 \pm 27.2 \mathrm{ng} / \mathrm{ml}$, thể tích $T \mathrm{TL}$ trung bình là $54.78 \pm 26.38 \mathrm{ml}$ ) được siêu âm qua đường trực tràng kết hợp đàn hồi mô và được sinh thiết 12 mẩu tại khoa Chẩn đoán hình ảnh của bệnh viện Hữu nghị Việt Đức.

Mỗi bệnh nhân được sinh thiết 12 mẫu và chia thành 4 vùng tổn thương tương ứng với vùng ngoại vi phải (mảnh số $1,2,3$ ), vùng chuyển tiếp phải (mảnh số 4,5,6), vùng chuyển tiếp trái (mảnh số $7,8,9)$, vùng ngoai vi trái (mảnh số 10 , $11,12)$. Mỗi vùng được đánh giá lần lượt trên siêu âm 2D, Doppler màu và $\mathrm{SE}$. Dựa vào tiêu chuẩn chẩn đoán UTTTL của mỗi kĩ thuật siêu âm đã nêu trên, mỗi vùng được ghi nhận là có hay không có tổn thương nghi ngờ ung thư đối với mỗi kĩ thuật siêu âm.

Phân tích 296 vùng có tất cả 78 tổn thương ung thư được xác định bằng giải phẫu bệnh, trong đó có 36 tổn thương ở vùng ngoại vi và 42 tổn thương ở vùng chuyển tiếp.

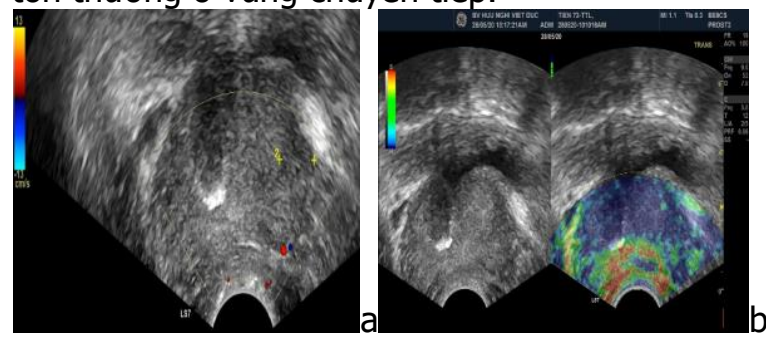

Hình 2: Bệnh nhân H.M.T 72 tuổi, PSA $64.3 \mathrm{ng} / \mathrm{ml}$, (a) tổn thương giảm âm không đồng nhất vùng chuyển tiếp trái, không tăng sinh mạch trên siêu âm Doppler, tổn thương tương ứng SE score 4 trên siêu âm đàn hồi (trung tâm màu xanh dương biểu hiện cứng, ngoại vi màu xanh lá biểu hiện mềm), gleason $4+3=7$.

Bảng 1 thể hiện giá trị của siêu âm 2D, 2D kết hợp Doppler màu và kết hợp 3 kĩ thuật trong chẩn đoán UTTTL ở vùng ngoại vi. Siêu ầm 2D có độ nhạy $72.2 \%$, độ đặc hiệu $67 \%$. Siêu âm 2D kết hợp Doppler màu có độ nhạy không đổi, độ đặc hiệu $66.1 \%$. Kết hợp cả ba kĩ thuậtlàm tăng độ nhạy và độ đặc hiệu lần lượt là $80.6 \%$ và $64.3 \%$.

Bảng 1: Giá trị của siêu âm 2D, 2D+Doppler màu, 2D+Doppler màu+SE trong chẩn đoán UTTTL ở vùng ngoại vi ( $n=148)$

\begin{tabular}{|c|c|c|c|c|c|}
\hline Kỹ thuật siêu âm & $\begin{array}{c}\text { Độ nhạy } \\
\mathbf{( \% )}\end{array}$ & $\begin{array}{c}\text { Độ đặc } \\
\text { hiệu (\%) }\end{array}$ & $\begin{array}{c}\text { Giá trị dự } \\
\text { đoán dương } \\
\text { tính (\%) }\end{array}$ & $\begin{array}{c}\text { Giá trị dự } \\
\text { đoán âm } \\
\text { tính (\%) }\end{array}$ & $\begin{array}{c}\text { Độ chính } \\
\text { xác (\%) }\end{array}$ \\
\hline 2D & 72.2 & 67 & 41.3 & 88.2 & 68.2 \\
\hline 2D+Doppler màu & 72.2 & 66.1 & 40.6 & 88.1 & 67.6 \\
\hline 2D+Doppler màu+SE & 80.6 & 64.3 & 42 & 91.1 & 90.1 \\
\hline
\end{tabular}

Bảng 2 thể hiện giá trị của siêu âm 2D, 2D kết hợp Doppler màuvà kết hợp cả ba kĩ thuật trong chẩn đoán UTTTL ở vùng chuyển tiếp. Siêu âm $2 \mathrm{D}$ có độ nhạy $40.5 \%$, độ đặc hiệu $88.7 \%$. Siêu âm 2D kết hợp Doppler màu có độ nhạy $66.7 \%$, độ đặc hiệu 59.4\%. Kết hợp cả ba kĩ thuật có độ nhạy $85.7 \%$, độ đặc hiệu $59.4 \%$.

Bảng 2: Giá trị của siêu âm 2D, 2D+Doppler màu, 2D+Doppler màu+SE trong chẩn đoán UTTTL ở vùng chuyển tiếp ( $n=148)$

\begin{tabular}{|c|c|c|c|c|c|}
\hline Kỹ thuật siêu âm & $\begin{array}{c}\text { Độ nhạy } \\
(\mathbf{\%})\end{array}$ & $\begin{array}{c}\text { Độ đặc } \\
\text { hiệu } \\
\mathbf{( \% )}\end{array}$ & $\begin{array}{c}\text { Giá trị dự } \\
\text { đoán dương } \\
\text { tính (\%) }\end{array}$ & $\begin{array}{c}\text { Giá trị dự } \\
\text { đoán âm } \\
\text { tính (\%) }\end{array}$ & $\begin{array}{c}\text { Độ chính } \\
\text { xác (\%) }\end{array}$ \\
\hline 2D & 40.5 & 88.7 & 58.6 & 79 & 75 \\
\hline 2D+Doppler màu & 66.7 & 59.4 & 39.4 & 81.8 & 61.5 \\
\hline 2D+Doppler màu+SE & 85.7 & 59.4 & 45.6 & 91.3 & 66.9 \\
\hline
\end{tabular}

Bảng 3 thể hiện giá trị của siêu âm 2D, 2D+Doppler màuvà kết hợp ba kĩ thuật trong chấn đoán UTTTL ở cả hai vùng. Siêu âm $2 \mathrm{D}$ có độ nhạy $55.1 \%$, độ đặc hiệu $77.5 \%$. Siểu âm $2 \mathrm{D}$ kết hợp Doppler màu có độ nhạy $69.2 \%$, độ đặc hiệu $62.8 \%$. Kết hợp ba kĩ thuật có độ nhạy $83.3 \%$, độ đặc hiệu $61.9 \%$. 
Bảng 3: Giá trị của siêu âm 2D, 2D+Doppler màu, 2D+Doppler màu+SE trong chẩn đoán UTTTL ở cả hai vùng ( $n=296)$

\begin{tabular}{|c|c|c|c|c|c|}
\hline Kỹ thuật siêu âm & $\begin{array}{c}\text { Độ nhạy } \\
(\mathbf{\%})\end{array}$ & $\begin{array}{c}\text { Độ đặc } \\
\text { hiệu (\%) }\end{array}$ & $\begin{array}{c}\text { Giá trị dư đoán } \\
\text { dướng tính } \\
(\mathbf{\%})\end{array}$ & $\begin{array}{c}\text { Giá trị dự } \\
\text { đoán âm } \\
\text { tính (\%) }\end{array}$ & $\begin{array}{c}\text { Độ chính } \\
\text { xác (\%) }\end{array}$ \\
\hline 2D & 55.1 & 77.5 & 46.7 & 82.8 & 71.6 \\
\hline 2D+Doppler màu & 69.2 & 62.8 & 40 & 85.1 & 64.5 \\
\hline 2D+Doppler màu+SE & 83.3 & 61.9 & 43.9 & 91.2 & 67.6 \\
\hline
\end{tabular}

\section{BÀN LUÂNN}

Phần lớn UTTTL có xu hướng tiển triển chậm, mức độ thấp và không quá ác tính. Tổn thương có thể phát triển ra ngoài bao tuyến hoặc khu trú trong tuyến qua nhiều thập $\mathrm{Ki}^{1}$. UTTTL chủ yếu nằm ở vùng ngoại vi $(70 \%)$ thuận lợi cho siêu âm qua đường trực tràng dể tiếp cận tổn thương ${ }^{3}$.

Trong nghiên cứu của chúng tôi, tỷ lệ ung thư chiếm 38\% (28/74), UTTTL thường gặp ở độ tuổi từ 55 đến 77 , thể tích TTL và PSA toàn phần trung bình trong nhóm ung thư lần lượt là 45.9 $\pm 24.7 \mathrm{ml}$ và $38.6 \pm 36 \mathrm{ng} / \mathrm{ml}$. Độ tuổi và tỷ lê ung thư trong mẫu nghiên cứu gẩn tương đồng với kết quả của Giurgiü. Thể tích TTL và PSA toàn phần trung bình của mẫu nghiên cứu cao hơn so với các nghiên cứu trước.

Nhu mô vùng ngoại vi bình thường có độ hồi âm đồng nhất và độ cứng trung gian do vậy một tổn thương ung thư nằm ở vùng ngoại vi có độ hồi âm thay đổi và có đô̂ cứng tăng sẽ dễ được phát hiện trên siêu âm. Ngược lại vùng chuyển tiếp vốn có độ hồi âm không đồng nhất trên siêu âm và có độ cứng tăng hơn nhất là ở người già khi có các nốt tăng sản, do vậy rất khó có thể phát hiện được các tổn thương khu trú tại vùng này ${ }^{3}$.

Trong nghiên cứu của chúng tôi, độ nhạy của siêu âm 2D, 2D+Doppler màu và 2D+Doppler màu+SE trong chẩn đoán UTTTL ở vùng ngoai vi lần lượt là $72.2 \%, 72.2 \%$ và $80.6 \%$; độ đặc hiệu lần lượt là $67 \%, 66.1 \%$ và $64.3 \%$. Như vậy khi kết hợp cả 3 kĩ thuật có làm tăng độ nhạy trong chẩn đoán UTTTL ở vùng ngoai vi. Theo nghiên cứu của Gadalla ${ }^{9}$ độ nhạy của SE trong chẩn đoán UTTTL ở vùng ngoại vi $100 \%$ và $60 \%$ tương ứng với vùng ngoại vi phải và trái. Nguyên nhân làm cho độ nhay của SE trong nghiên cứu của chúng tôi thấp hơn là do SE hạn chế trong việc phát hiện những tổn thương ung thư nhỏ và có điểm Gleason thấp ${ }^{5}$. Trong nghiên cứu của chúng tôi SE chỉ phát hiện được $2 / 5$ tổn thương có điểm Gleason 6 và 3/7 tổn thương có điểm Gleason 7.

Đối với những tổn thương ung thư ở vùng chuyển tiếp, độ nhạy của siêu âm $2 \mathrm{D}$ chỉ là $40.5 \%$ do đặc tính không đồng nhất vốn có của vùng chuyển tiếp. Thể tích TTL trung bình của mẫu nghiên cứu cao hơn so với các nghiên cứu trước nên đây cũng là nguyên nhân làm giảm độ nhay của siêu âm do vùng chuyển tiếp định vị ở phía trước tuyến nên khi thể tích TTL càng tăng thì khả năng khảo sát của sóng siêu âm càng

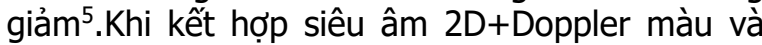
$2 \mathrm{D}+$ Doppler màu+SE có độ nhạy tăng lên lần lượt là $66.7 \%$ và $85.7 \%$ nhưng độ đặc hiệu giảm còn $59.4 \%$.

Khi đánh giá tổn thương trên cả hai vùng chuyển tiếp và ngoại vi, độ nhạy của siêu âm $2 \mathrm{D}$ chỉ $55.1 \%$ do trong mấu nghiền cứu của chúng tôi phần lớn tổn thương ung thư định vị ở vùng chuyển tiếp (42/78 tổn thương). Khi kết hợp hai và ba phương pháp độ nhạy tăng lên lần lượt là $69.2 \%$ và $83.3 \%$. Theo nghiên cứu của Giurgiu ${ }^{8}$, độ nhạy của siêu âm 2D, 2D+Doppler, 2D+Doppler+SE lần lượt là $67.05 \%, 78.57 \%$, $89.2 \%$; độ đặc hiêuu lần lượt là $51.4 \%, 27.02 \%$ và $24.32 \%$. Nghiển cứu của chúng tôi có độ nhạy thấp hơn nhưng độ đặc hiệu cao hơn so với nghiên cứu của Giurgiư ${ }^{8}$.

Hạn chế của nghiên cứu là do siêu âm đàn hồi mô sử dụng các thông số bán định lượng và là kĩ thuật phụ thuộc vào kinh nghiệm của người làm. Ngoài ra cấu tạo của đầu dò trực tràng có khoảng cách giữa hai bình diện rộng nên mặt phẳng tiếp cận với thành trực tràng không đều.

\section{KẾT LUẦN}

Qua nghiên cứu chúng tôi nhận thấy siêu âm đa phương tiện qua đường trực tràng gồm các kĩ thuật 2D, Doppler, SE có giá trị thay đổi khi đánh giá tổn thương nằm ở vùng ngoại vi hay chuyển tiếp. Siêu âm 2D qua đường trực tràng có độ nhạy cao trong chẩn đoán UTTTL nằm ở vùng ngoại vi nhưng có độ nhay thấp trong chẩn đoán UTTTL nằm ở vùng chuyển tiếp. Kết hợphai hoặc ba kĩ thuật siêu âm đều làm tăng độ nhạy trong chẩn đoán UTTTL đặc biệt ở vùng chuyển tiếp. Siêu âm đàn hồi mô được coi là phương pháp bồ trợ của siêu âm TTL qua đường trực tràng làm tăng độ nhạy chẩn đoán UTTTL.

\section{TÀI LIÊU THAM KHẢO}

1. Leslie SW, Soon-Sutton TL, Sajjad H, Siref LE. Prostate Cancer. In: StatPearls. StatPearls 
Publishing; 2021. Accessed September 26, 2021. http://www.ncbi.nlm.nih.gov/books/NBK470550/

2. Barr, Richard G (2017). Elastography a Practical : "Elastography of the Prostate", 87-99.

3. Harvey CJ, Pilcher J, Richenberg J, Patel U, Frauscher F. Applications of transrectal ultrasound in prostate cancer. $\mathrm{Br} \mathrm{J}$ Radiol. 2012;85(Spec Iss 1):S3-S17. doi:10.1259/bjr/56357549

4. Tangel MR, Rastinehad AR. Advances in prostate cancer imaging. F1000Res. 2018;7:1337. doi:10.12688/f1000research.14498.1

5. Barr RG, Cosgrove $D$, Brock $M$, et al. WFUMB Guidelines and Recommendations on the Clinical Use of Ultrasound Elastography: Part 5. Prostate. Ultrasound in Medicine \& Biology. 2017;43(1):2748. doi:10.1016/j.ultrasmedbio.2016.06.020

6. Dietrich CF, Barr RG, Farrokh A, et al. Strain
Elastography - How To Do It? Ultrasound Int Open. 2017;3(4):E137-E149. doi:10.1055/s-0043119412

7. Kamoi K, Okihara K, Ochiai A, et al. The Utility of Transrectal Real-Time Elastography in the Diagnosis of Prostate Cancer. Ultrasound in Medicine and Biology. 2008;34(7):1025-1032. doi:10.1016/j.ultrasmedbio.2007.12.002

8. Giurgiu CR, Manea $C$, Crişan N, Bungărdean C, Coman I, Dudea SM. Real-time sonoelastography in the diagnosis of prostate cancer. Med Ultrason. 2011;13(1):5-9.

9. El Fattah Hassan Gadalla AA, El Rahman SFA Anis SE, El-Sayed khalil M. Value of ultrasound elastography versus transrectal prostatic biopsy in prostatic cancer detection. The Egyptian Journal of Radiology and Nuclear Medicine. 2015;46(3):761768. doi:10.1016/j.ejrnm.2015.05.009

\section{ĐĂC ĐIỂM KỸ THUÂTT, TÍNH AN TOÀN CỦA NộI SOI BÓNG ĐƠN Ở BÊ̂NH NHÂN CHẢY MÁU TIÊU HÓA NGHI Ở RUỘT NON}

\section{TÓM TẮT}

Mục tiêu: Mô tả đặc điểm kỹ thuật và tính an toàn của nội soi bóng đợn ở bệnh nhân nghi chảy máu tiêu hóa (CMTH) ở ruột non. Đối tượng và phương pháp: Có 89 bệnh nhần nghi CMTH tại ruột non đước đưa vào nghiên cứu. Trước khi thực hiện nội soi ruột non bóng đơn (NSRNBĐ), tất cả các bềnh nhân đều được nôi soi dạ dày-tá tràng và đại tràng, nhưng khổng phát hiện thấy tổn thương gây CMTH. Các thông số theo dõ̃i: Đương soi, thời gian, chiều dài ruột non soi được và biến chứng. Kết quả: Tỷ lệ soi theo đường miệng, đường hậu môn và cả 2 đường, tương ứng là: $35,9 \%, 14,6 \%$ và $49,5 \%$. Thời gian trung bình (phút) theo đường miệng, đường hậu môn và cả hai đường, tương ứng là: $95,31 \pm 40,42 ; 51,92 \pm 29,69$ và $161,70 \pm 16,46$. Chiêuu dài trung bình (mét) của ruột non nội soi qua đường miệng, đường hậu môn và cả hai đường chiếm tỷ lệ tương ứng là: $2,49 \pm 0,94$; $1,32 \pm 0,74$ và $2,94 \pm 1,26$. Biến chứng hay gặp sau NSRNBĐ là viêm tụy cấp mức độ nhẹ: 3/89 bẹnh nhân $(3,4 \%)$. Kết luận: Nội soi bóng đơn là một kỹ thuật an toàn, có hiệu trong chẩn đoán bệnh lý tại ruột non.

Tư khóa: Chảy máu tiêu hóa, nội soi ruột non bóng đơn

\section{SUMMARY}

${ }^{1}$ Bênh viên Bach Mai

²Bệnh viện TWQÐ

${ }^{3}$ Bệnh viện 103-HVQY

Chịu trách nhiệm chính: Đỗ Anh Giang

Email: dranhgiangbm@gmail.com

Ngày nhận bài: 6.8.2021

Ngày phản biện khoa học: 6.10 .2021

Ngày duyệt bài: 15.10.2021
Đỗ Anh Giang'1 Vũ Văn Khiên' Phạm Thị Thu Hồ ${ }^{1}$, Dương Quang Huy ${ }^{3}$

\section{CHARACTERISTICS AND SAFETY OF SINGLE BALOON ENTEROSCOPY IN PATIENTS WITH \\ SUSPECTED IN SMALL BOWEL BLEEDING}

Aim: Characteristics and safety of single balloon enteroscopy (SBE) in patients with suspected in smal bowel bleeding. Patient and methods: There were 89 patients with suspected in small bowel bleeding in the study. Before performing SBE, all patients underwent upper GI endoscopy and colonoscopy, but no lesions were detected. Follow-up parameters: endoscopic path, length of endoscopic small intestine, endoscopic time and complications. Results: The percentages of oral, anal and both routes were $35.9 \%, 14.6 \%$ and $49.5 \%$, respectively. Mean time (minutes) by oral, anal and both routes were $95.31 \pm$ $40.42 ; 51.92 \pm 29.69$ and $161.70 \pm 16.46$, respectively. The mean length (meters) of the small intestine through endoscopic oral, anal and both routes were $2.49 \pm 0.94 ; 1.32 \pm 0.74$ and $2.94 \pm$ 1.26 , respectively. The common complication after SBE is mild acute pancreatitis: $3 / 89$ patients (3.4\%). Conclusion: Single-balloon endoscopy is a safe technique in the diagnosis of patients with suspected small bowel bleeding.

Key words: GI bleeding, single balloon enteroscopy

\section{I. ĐĂT VẤN ĐỀ}

Trong các thập kỷ trước, CMTH tại ruột non vẫn là một cơ quan "bí hiểm", vì khồng có các thiết bị hiện đại thăm dò trực tiếp tại ruột, giúp chẩn đoán chính xác bệnh. Các biện pháp chẩn đoán hình ảnh khác như chụp cộng hưởng từ ồ bụng, chụp cắt lớp vi tính ổ bụng, chụp xạ hình ổ bụng và kể cả nội soi viên nang... cũng chỉ là các phương pháp trợ giúp cho chẩn đoán cho bệnh 